\title{
SURVEYS OF RADIO SOURCES (DISCUSSION)
}

General discussion following the group of survey papers presented by DE BRUYN, BECKER, COTTON, WRIGHT (p. 495, 499, 503, 507)

Ekers: How complete are your surveys to extended and multiple component sources.

Wright: I think the best way of ensuring good completeness for extended sources is to survey for them using a large single-dish and get structure for them with an array. This just happens to be the way the PMN work was done and is proceeding!

Becker: Comparison between $\log N$ - $\log S$ plots for the FIRST catalogue and published data suggest that the FIRST survey is complete at the $\sim 95 \%$ level.

de Bruyn: I do not think we could miss sources because they are too big. However, we could fail to properly recognize doubles or multiples (on $3^{\prime}-15^{\prime}$ scales) and the catalogue could not possibly be complete because of this. There will be a warning in the catalogue if sources are blended. 'Blind' analysis of a catalogue is therefore not without risk.

Cotton: The NVSS should not miss too many sources but associating separated regions of emission with individual sources may be difficult especially in the source catalog. At present association of regions needs to be done by eye from the images. If the source had significant residuals this is marked in the catalog indicating more complex or extended structure.

Condon: Not only does resolution cause sources to disappear form a survey catalogue, it will cause the integrated flux densities of extended catalogued sources to be too low. Users selecting flux-limited samples should watch out for incompleteness at any cut off level, even one well above the nominal catalog limit. This problem is smallest for low-resolution single dish surveys, slightly greater for the WENSS (which has good $(u, v)$-plane coverage), and worst for the VLA snapshot surveys (which have poor $(u, v)$ - coverage). 
Machalsky: Will your observation data be available to international community? If yes, how and when?

Becker: Yes. The images are available by anonymous ftp from NRAO. They are also in the STScI archival system. The catalog is available through WWW at http://sundog.stsci.edu

Jauncey On behalf of VSOP Survey Working Group I would like to note the directed high brightness temperature survey to be undertaken by the VSOP SWG. The aim is to look for high $T_{b}$ components in a complete all-sky sample of flat-spectrum sources found at $5 \mathrm{Ghz}$ and satisfying $S_{5} \geq 1 J y, \alpha \leq 0.5,|b| \geq 10^{\circ}$. More details to be found in the VSOP poster and in the VSOP AO.

Hunstead: Comment: The Molonglo Observatory Synthesis Telescope (MOST), operated by the University of Sydney, will be carrying out a survey on the southern sky at $843 \mathrm{MHz}$ south of $-30^{\circ}$ beginning $\sim$ mid 1996 and lasting $5-10$ years. The MOST has a synthesised beamwidth of $43^{\prime \prime}$ and a positional accuracy $\sim 1^{\prime \prime}$. With the new field size of $2^{\circ} .7$ and upgraded receivers the expected thermal noise level $(1 \sigma)$ is $1 \mathrm{mJy}$ in a 12-hour observation. It is anticipated that over 400000 sources will be detected down to limits $\sim 5 \mathrm{mJy}$. Further information may be obtained from most@physics.usyd.edu.au

Jauncey: Two major advantages of large surveys are: a) High statistical value of large numbers, and b) ability to find rare and unusual new objects. Both of these can be compromised in the data handling etc, by building in biases. An example of this is the colour/morphology selection for quasar indents. There are strong colour- $z$ correlations that show that excluding red stellar idents are often of great interest. 